Communications in Physics, Vol.27, No. 1 (2017), pp. 55-64

DOI:10.15625/0868-3166/27/1/8912

\title{
X-RAY ABSORPTION FINE STRUCTURE OF BCC CRYSTALS STUDIED BASED ON HIGH-ORDER EXPANDED DEBYE-WALLER FACTORS
}

\author{
NGUYEN VAN HUNG ${ }^{1, \dagger}$, TRINH THI HUE ${ }^{1}$, HA DANG KHOA ${ }^{2}$ AND TONG SY TIEN ${ }^{3}$ \\ ${ }^{1}$ Department of Physics, Hanoi University of Science. 334 Nguyen Trai, Thanh Xuan, Hanoi, \\ Vietnam \\ ${ }^{2}$ School of Engineering Physics, Hanoi University of Science and Technology, \\ 1 Dai Co Viet, Hai Ba Trung, Hanoi, Vietnam \\ ${ }^{3}$ Department of Basic Sciences, University of Fire Fighting \& Prevention, \\ 243 Khuat Duy Tien, Thanh Xuan, Hanoi, Vietnam \\ ${ }^{\dagger}$ E-mail: hungnv@vnu.edu.vn
}

Received 24 November 2016

Accepted for publication 30 March 2017

\begin{abstract}
In this work, the X-ray absorption fine structure (XAFS) spectra of bcc crystals and their Fourier transform magnitudes have been studied based on the anharmonic correlated Debye model high-order expanded Debye-Waller factors. The many-body effects are taken into account in present one-dimensional model based on the anharmonic effective potential that includes interactions of absorber and backscatterer atoms with their first shell near neighbors, where the Morse potential is assumed to describe the single-pair atomic interaction. Analytical expressions of four first temperature-dependent cumulants of bcc crystals have been derived using the manybody perturbation approach. The obtained cumulants are applied to calculating XAFS spectra and their Fourier transform magnitudes. Numerical results for bcc phase of Fe are found to be in good agreement with the experimental data.
\end{abstract}

Keywords: Debye-Waller factor, effective potential, correlated Debye model, XAFS, bcc crystals. Classification numbers: 61.05.C, 61.66.Bi, 78.70.Dm.

\section{INTRODUCTION}

The X-ray Absorption Fine Structure (XAFS) spectra have developed into a powerful technique for providing information on the local atomic structure and thermal effects of substances [120]. The formalism for including anharmonic effects in XAFS is often based on the cumulant expansion [1] where the even cumulants contribute to the amplitude, the odd ones contribute to the (C) 2017 Vietnam Academy of Science and Technology 
phase of XAFS spectra, and for small anharmonicities, it is sufficient to keep the third and fourth cumulants [2]. The accurate Debye-Waller factors (DWFs) varying as $e^{-W(T)}$ with temperature $T$ and wave number $k$ (or energy) due to their exponential damping are crucial to quantitative treatment of XAFS spectra. Consequently, the lack of the precise DWFs has been one of the biggest limitations to accurate structural determinations (e.g., the coordination numbers and the atomic distances) from XAFS experiments.

Many efforts have been made to develop procedures for the calculation and analysis of XAFS cumulants using the classical theories [7-9] and quantum theories [1-6, 10-20]. Unfortunately, there is still no theoretical result on XAFS of bcc crystals while their experimental results are available [21].

This work is a theoretical study of XAFS spectra and their Fourier transform magnitudes of bcc crystals using the DWFs presented in terms of cumulant expansion up to the fourth order based on the anharmonic correlated Debye model (ACDM) [20]. In Sec. II, the analytical expressions have been presented for four first temperature-dependent cumulants of bcc crystals derived using the many-body perturbation approach (MBPA) [22], the dispersion relation and the anharmonic effective potential parameters obtained based on the first shell near neighbor contribution approach (FSNNCA), where the Morse potential is assumed to describe the single-pair atomic interaction. The obtained cumulants are applied to calculating XAFS spectra and their Fourier transform magnitudes. Numerical results for bcc phase of Fe are compared with the experimental data in [21] which show a good agreement.

\section{FORMALISM}

The formalism for including anharmonic effects in XAFS is often based on the cumulant expansion approach [1] from which the expression for anharmonic XAFS spectra has the form

$$
\chi(k)=F(k) \frac{e^{-2 R / \lambda(k)}}{k R^{2}} \operatorname{Im}\left\{e^{i \Phi(k)} \exp \left[2 i k R+\sum_{n} \frac{(2 i k)^{n}}{n !} \sigma^{(n)}\right]\right\},
$$

where $F(k)$ is the real atomic backscattering amplitude, $\mathrm{k}$ and $\lambda$ are the wave number and the mean free path of photoelectron, respectively, $\Phi$ is the net phase shift, $R=\langle r\rangle$ with $r$ being the instantaneous bond length between absorber and backscatterer atoms, and $\sigma^{(n)}(n=1,2,3,4, \ldots)$ are the cumulants describing the high-order expanded DWFs.

If limiting to the fourth cumulant, the temperature $T$ - and wave number k-dependent anharmonic contributions to the amplitude $F_{A}(k, T)$ and to the phase $\Phi_{A}(k, T)$ of XAFS spectra are given by

$$
\begin{aligned}
F_{A}(k, T) & =\exp \left(-\frac{2}{3} k^{4} \sigma^{(4)}(T)\right), \\
\Phi_{A}(k, T) & =-\frac{4 k \sigma^{2}(T)}{R}\left(1+\frac{R}{\lambda}\right)-\frac{4}{3} k^{3} \sigma^{(3)}(T),
\end{aligned}
$$

Hence, for calculating XAFS spectra and their Fourier transform magnitudes of bcc crystals using Eqs. (1) and (2), it is necessary to calculate their DWFs expanded up to the fourth order.

In order to include the anharmonic effects, the Hamiltonian of system is written in the summation of the harmonic and anharmonic components, $H_{0}$ and $H_{a}$, respectively 


$$
H=H_{0}+H_{a}, H_{a}=H_{c}+H_{q},
$$

where the anharmonic component $H_{a}$ contains the cubic $H_{c}$ and the fourth order $H_{q}$ terms including the anharmonic interatomic effective potential parameters of bcc crystals.

The anharmonic interatomic effective potential expanded up to the fourth order in the present theory for bcc crystals is expressed as a function of the displacement $x=r-r_{0}$ along the bond direction with $r$ and $r_{0}$ being the instantaneous and equilibrium distances between absorber and atoms, respectively

$$
V_{e f f}(x) \approx \frac{1}{2} k_{e f f} x^{2}+k_{3 e f f} x^{3}+k_{4 e f f} x^{4}
$$

where $k_{e f f}$ is the effective local force constant, $k_{3 e f f}$ and $k_{4 e f f}$ are the anharmonic parameters giving an asymmetry of the anharmonic effective potential.

For bcc crystals each atom is bonded to 8 near neighbors. Then, the effective potential given by Eq. (4) defined based on the FSNNCA has the form

$$
V_{e f f}(x)=V(x)+2 V\left(-\frac{x}{2}\right)+6 V\left(\frac{x}{6}\right)+6 V\left(-\frac{x}{6}\right),
$$

which is the sum over not only the term $\mathrm{V}(\mathrm{x})$ describing the pair-interaction between absorber and backscatterer atoms but also the other terms describing the projections of their pair-interactions with 14 first shell near neighbors of bcc crystals along the bond direction excluding the absorber and backscatterer themselves whose contributions are already described by $\mathrm{V}(\mathrm{x})$.

Applying the Morse potential expanded up to the fourth order as

$$
\begin{aligned}
V(x) & =D\left(e^{-2 \alpha x}-2 e^{-\alpha x}\right) \\
& \approx D\left(-1+\alpha^{2} x^{2}-\alpha^{3} x^{3}+\frac{7}{12} \alpha^{4} x^{4}\right)
\end{aligned}
$$

to Eq. (5) and comparing the result to Eq. (4), we obtain the values of $k_{e f f}, k_{3 e f f}, k_{4 e f f}$ for bcc crystals in terms of Morse potential parameters

$$
k_{\text {eff }}=\frac{11}{3} D \alpha^{2}, k_{3 e f f}=-\frac{3}{4} D \alpha^{3}, k_{4 e f f}=\frac{1715}{2592} D \alpha^{4},
$$

where $\alpha$ describes the width of the potential and $D$ is dissociation energy.

Hence, the anharmonic effective potential for bcc crystals in Eq. (5) has the form

$$
V_{e f f}(x) \approx \frac{11}{6} D \alpha^{2} x^{2}-\frac{3}{4} D \alpha^{3} x^{3}+\frac{1715}{2592} D \alpha^{4} x^{4}
$$

Note that the above mentioned lattice contributions based on the FSNNCA, to oscillation between absorber and backscatterer atoms described by the projections of their pair-interactions with 14 first shell near neighbors along the bond direction, make it possible to take into account the many-body effects in the present one-dimensional model for bcc crystals.

The derivation of present ACDM for bcc crystals using the MBPA [22] is based on the dualism of an elementary particle in quantum theory, its corpuscular and wave properties. Then, we can describe the system in Debye model involving all different frequencies up to the Debye frequency as a system consisting of many bodies or many phonons each of which corresponds to a wave having frequency $\omega(q)$ and wave number $q$ varied in the first Brillouin zone (BZ). 
For this purpose, the displacement $u_{n}^{\prime} s$ in the parameter $x$ in terms of the displacement of $n$th atom $u_{n}$ of the one dimensional chain is described by

$$
x_{n}=u_{n+1}-u_{n}
$$

where the displacement $u_{n}$ is related to the phonon displacement operators $A_{q}$ [23] in the form

$$
\begin{aligned}
u_{n} & =\sqrt{\frac{\hbar}{2 N M}} \sum_{q} \frac{e^{i q a n}}{\sqrt{\omega(q)}} A_{q}, \\
A_{q} & =A_{-q}^{+}, \quad\left[A_{q}, A_{q^{\prime}}\right]=0 .
\end{aligned}
$$

Therefore, the value of $x_{n}$ in Eq. (9) is given by

$$
\begin{aligned}
x_{n} & =\sum_{q} e^{i q a n} f(q) A_{q}, \\
f(q) & =\sqrt{\frac{\hbar}{2 N M \omega(q)}}\left(e^{i q a}-1\right),
\end{aligned}
$$

where $N$ is the atomic number, $M$ is the mass of composite atoms and $a$ is the lattice constant.

The frequency $\omega(q)$ contained in Eq. (11) and then in all cumulant expressions derived for the vibration between absorber and backscatterer atoms in XAFS process describes the dispersion relation. Using the local force constant of the first equation of Eqs. (7), it has the form

$$
\omega(q)=2 \alpha \sqrt{\frac{11 D}{3 M}}\left|\sin \left(\frac{q a}{2}\right)\right|,|q| \leq \frac{\pi}{a}
$$

Using the above results in the MBPA [22], we have derived the analytical expressions for XAFS DWFs presented in terms of cumulant expansion up to the fourth order for bcc crystals.

The first cumulant describing the net thermal expansion or the lattice disorder in the XAFS theory has resulted as

$$
\begin{aligned}
& \sigma^{(1)}(T)=\langle x\rangle=\sigma_{0}^{(1)} \int_{0}^{\pi / a} \omega(q) \frac{1+Z(q)}{1-Z(q)} d q=\frac{\sigma_{0}^{(1)}}{\sigma_{0}^{2}} \sigma^{2}, \\
& \sigma_{0}^{(1)}=\frac{81 a \hbar}{484 \pi D \alpha}, Z(q)=\exp (\beta \hbar \omega(q)), \beta=\frac{1}{k_{B} T} .
\end{aligned}
$$

Here, $\sigma^{2}$ is the second cumulant describing the mean square relative displacement (MSRD) and has the following form

$$
\sigma^{2}(T)=\left\langle x^{2}\right\rangle=\sigma_{0}^{2} \int_{0}^{\pi / a} \omega(q) \frac{1+z(q)}{1-z(q)} d q, \quad \sigma_{0}^{2}=\frac{3 \hbar a}{22 \pi D \alpha^{2}}
$$


The third cumulant is the mean cubic relative displacement (MCRD) describing the asymmetry of the pair distribution function in the XAFS theory and is expressed as

$$
\begin{aligned}
& \sigma^{(3)}(T) \cong\left\langle x^{3}\right\rangle-3\left\langle x^{2}\right\rangle\langle x\rangle \\
& =\sigma_{0}^{(3)} \int_{0}^{\pi / a} d q_{1} \int_{-\pi / d}^{\pi / a-q_{1}} d q_{2} \frac{\omega\left(q_{1}\right) \omega\left(q_{2}\right) \omega\left(q_{1}+q_{2}\right)}{\omega\left(q_{1}\right)+\omega\left(q_{2}\right)+\omega\left(q_{1}+q_{2}\right)} \\
& \quad \times\left\{1+6 \frac{\omega\left(q_{1}\right)+\omega\left(q_{2}\right)}{\omega\left(q_{1}\right)+\omega\left(q_{2}\right)-\omega\left(q_{1}+q_{2}\right)} \frac{e^{\beta \hbar\left[\omega\left(q_{1}\right)+\omega\left(q_{2}\right)\right]}-e^{\beta \hbar \omega\left(q_{1}+q_{2}\right)}}{\left(e^{\beta \hbar \omega\left(q_{1}\right)}-1\right)\left(e^{\beta \hbar \omega\left(q_{2}\right)}-1\right)\left(e^{\beta \hbar \omega\left(q_{1}+q_{2}\right)}-1\right)}\right\}, \\
& \sigma_{0}^{(3)}=\frac{114 \times 10^{-4} \hbar^{2} a^{2}}{\pi^{2} D^{2} \alpha^{3}} .
\end{aligned}
$$

The fourth cumulant describes the anharmonic contribution to the XAFS amplitude and has the form

$$
\begin{aligned}
& \sigma^{(4)}(T) \cong\left\langle x^{4}\right\rangle-3\left\langle x^{2}\right\rangle^{2} \\
& =\sigma_{0}^{(4)} \int_{0}^{\pi / a} d q_{1} \int_{0}^{\pi / a-q_{1}} d q_{2} \int_{-\pi / a}^{\pi / a-\left(q_{1}+q_{2}\right)} d q_{3} \frac{\omega\left(q_{1}\right) \omega\left(q_{2}\right) \omega\left(q_{3}\right) \omega\left(q_{4}\right)}{\omega\left(q_{1}\right)+\omega\left(q_{2}\right)+\omega\left(q_{3}\right)+\omega\left(q_{4}\right)} \\
& \quad \times\left\{1+8 \frac{Z\left(q_{1}\right) Z\left(q_{2}\right) Z\left(q_{3}\right)-Z\left(q_{4}\right)}{\left(Z\left(q_{1}\right)-1\right)\left(Z\left(q_{2}\right)-1\right)\left(Z\left(q_{3}\right)-1\right)\left(Z\left(q_{4}\right)-1\right)} \frac{\omega\left(q_{1}\right)+\omega\left(q_{2}\right)+\omega\left(q_{3}\right)}{\omega\left(q_{1}\right)+\omega\left(q_{2}\right)+\omega\left(q_{3}\right)-\omega\left(q_{4}\right)}\right. \\
& \left.\quad+6 \frac{Z\left(q_{1}\right) Z\left(q_{2}\right)-Z\left(q_{3}\right) Z\left(q_{4}\right)}{\left(Z\left(q_{1}\right)-1\right)\left(Z\left(q_{2}\right)-1\right)\left(Z\left(q_{3}\right)-1\right)\left(Z\left(q_{4}\right)-1\right)} \frac{\omega\left(q_{3}\right)+\omega\left(q_{4}\right)}{\omega\left(q_{1}\right)+\omega\left(q_{2}\right)-\omega\left(q_{3}\right)-\omega\left(q_{4}\right)}\right\}, \\
& \sigma_{0}^{(4)}=\frac{165 \times 10^{-4} \hbar^{3} a^{3}}{2 \pi^{3} D^{3} \alpha^{4}}, q_{4}=-\left(q_{1}+q_{2}+q_{3}\right) .
\end{aligned}
$$

Note that in the above expressions for the cumulants of bcc crystals, $\sigma_{0}^{(1)}, \sigma_{0}^{2}, \sigma_{0}^{(3)}, \sigma_{0}^{(4)}$ are zero-point energy contributions to the first, second, third and fourth cumulant, respectively, and these cumulant expressions have been obtained for the case of large atomic number $\mathrm{N}$, when the summation over $q$ is replaced by the corresponding integral in the first BZ. Hence, the obtained cumulants described in Eqs. (13) - (16) contribute to the XAFS spectra of bcc crystals given by Eq. (1) containing the anharmonic contributions described by Eqs. (2).

\section{NUMERICAL RESULTS AND DISCUSSIONS}

Now the expressions derived in the previous section are applied to numerical calculations for bcc phase of Fe using its Morse potential parameters as follows: $D=0.418 \mathrm{eV}, \alpha=$ $1.397 \AA^{-1}[24]$.

Figures 1.a,b and 2.a,b show the dependences of the cumulants $\sigma^{(1)}, \sigma^{2}, \sigma^{(3)}$ and $\sigma^{(4)}$ on temperature for bcc phase of Fe calculated by the present theory. Our calculated results are in very good agreement with the experimental data in [21]. 

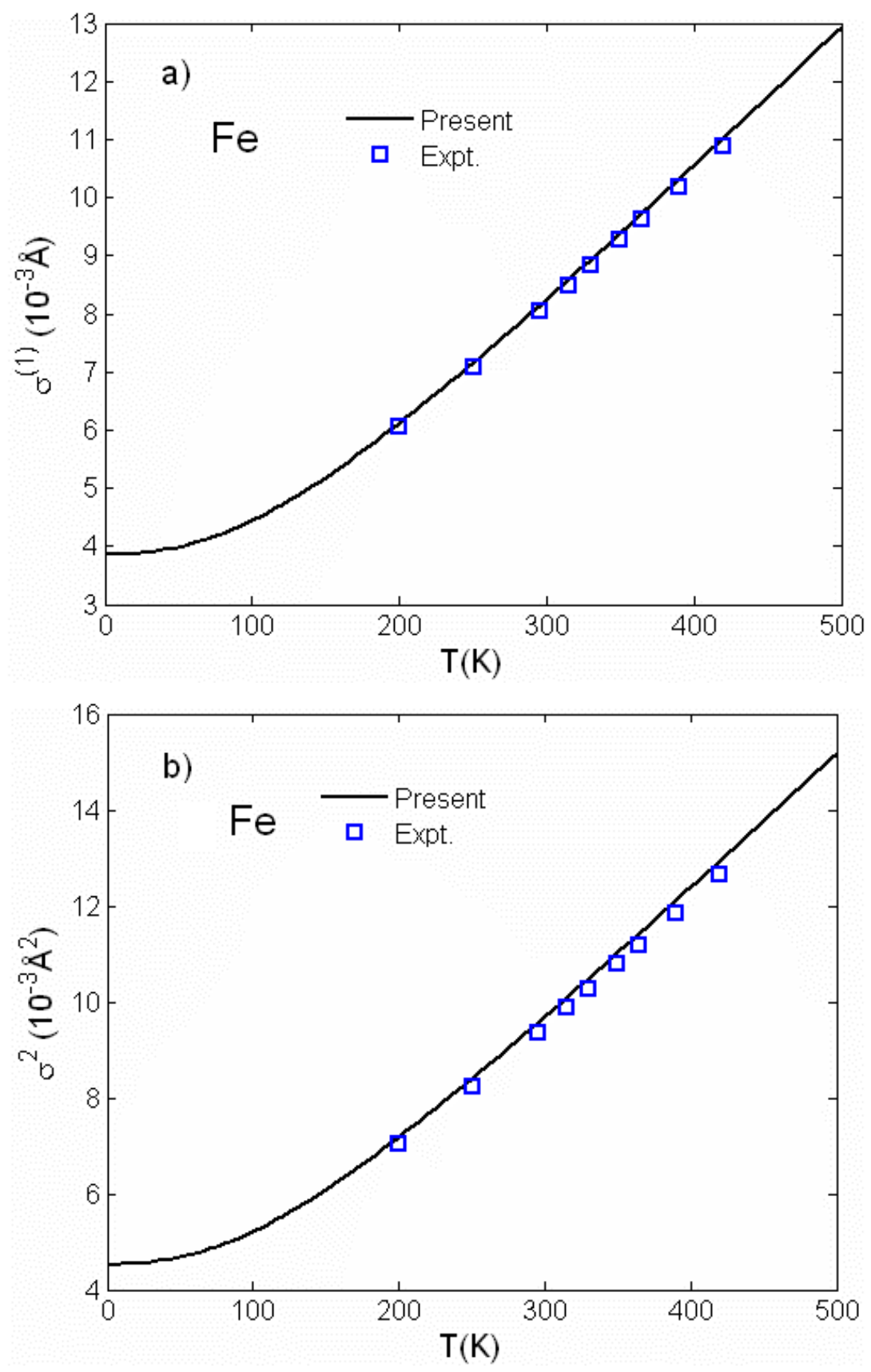

Fig. 1. The dependences of a) the first cumulant $\sigma^{(1)}(T)$ and b) the second cumulant $\sigma^{2}(\mathrm{~T})$ on temperature for bcc phase of Fe calculated by the present theory in the comparison with the experimental data in [21]. 

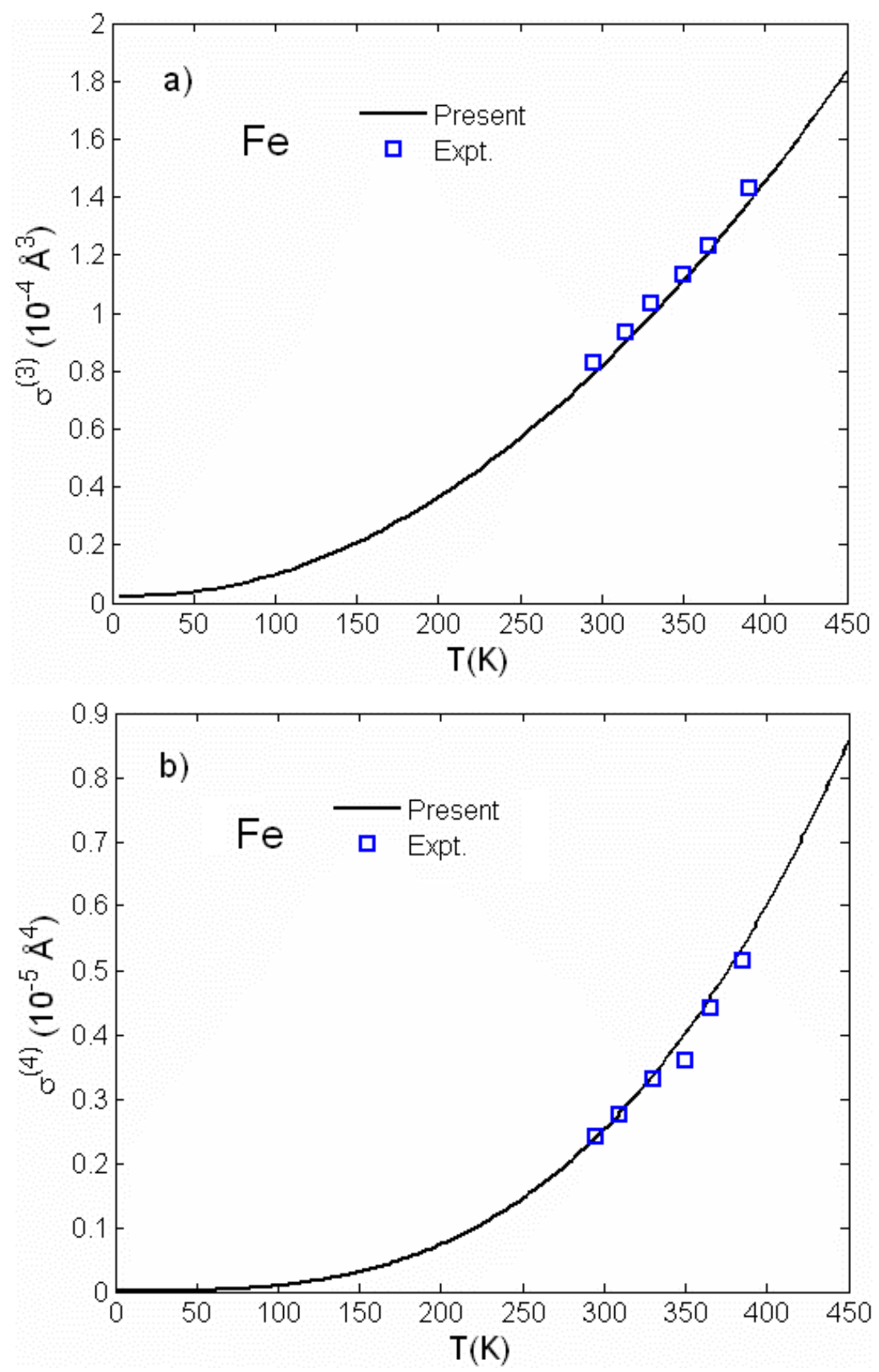

Fig. 2. The dependences of a) the third cumulant $\sigma^{(3)}(T)$ and b) the fourth cumulant $\sigma^{(4)}(T)$ on temperature for bcc phase of Fe calculated by the present theory in the comparison with the experimental data in [21]. 
Figures 3.a,b describe the dependence of anharmonic contributions $F_{A}(T, k)$ and $\Phi_{A}(T, k)$ to the XAFS spectra amplitude and phase on temperature and wave number for bcc phase of $\mathrm{Fe}$ calculated by the present theory. The obtained results show that when the temperature and the wave number increase, these anharmonic contributions also increase.
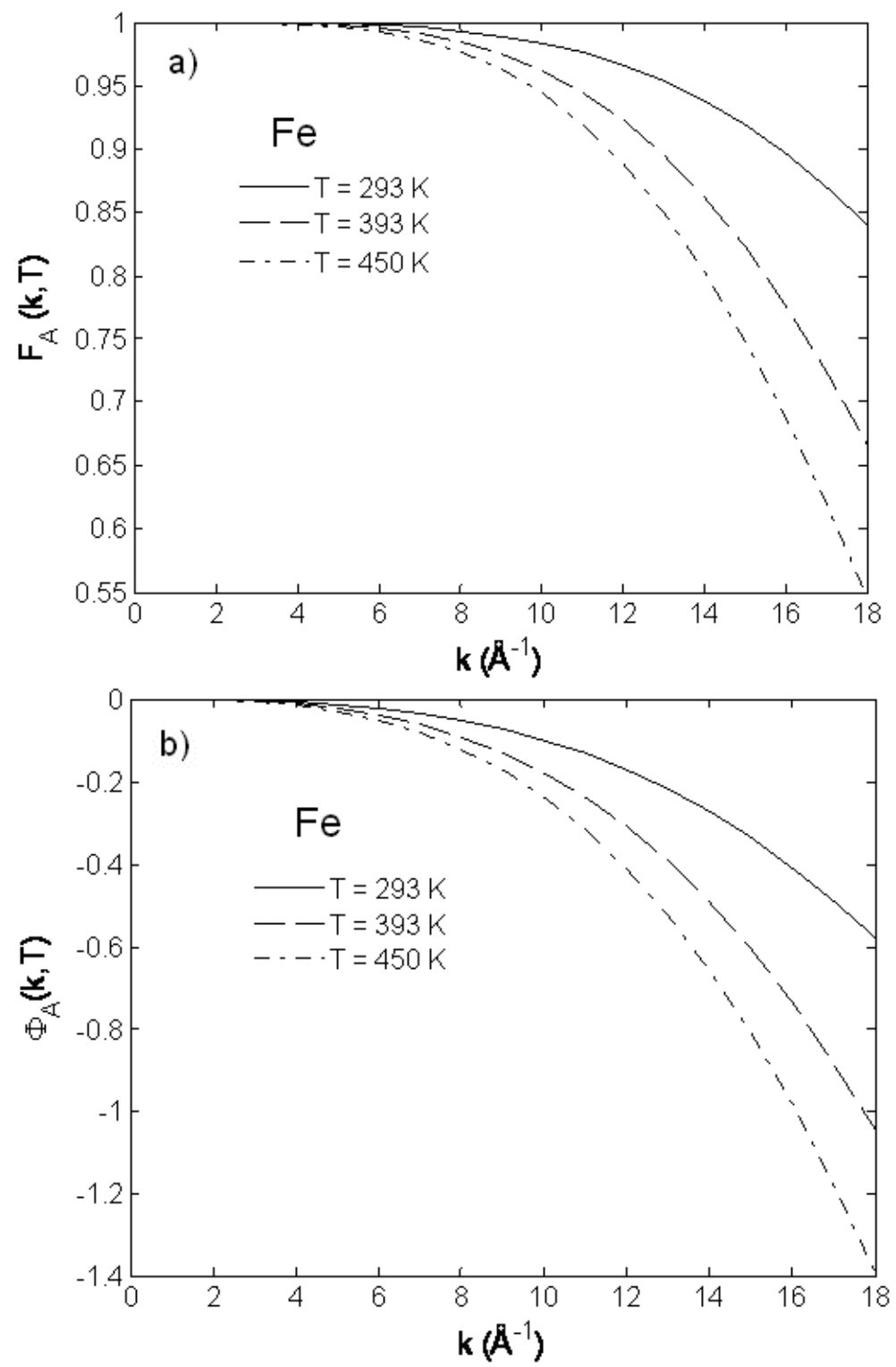

Fig. 3. The dependence of anharmonic contributions a) $F_{A}(T, k)$ and b) $\Phi_{A}(T, k)$ to the XAFS spectra amplitude and phase on temperature and wave number for bcc phase of $\mathrm{Fe}$ calculated by the present theory. 

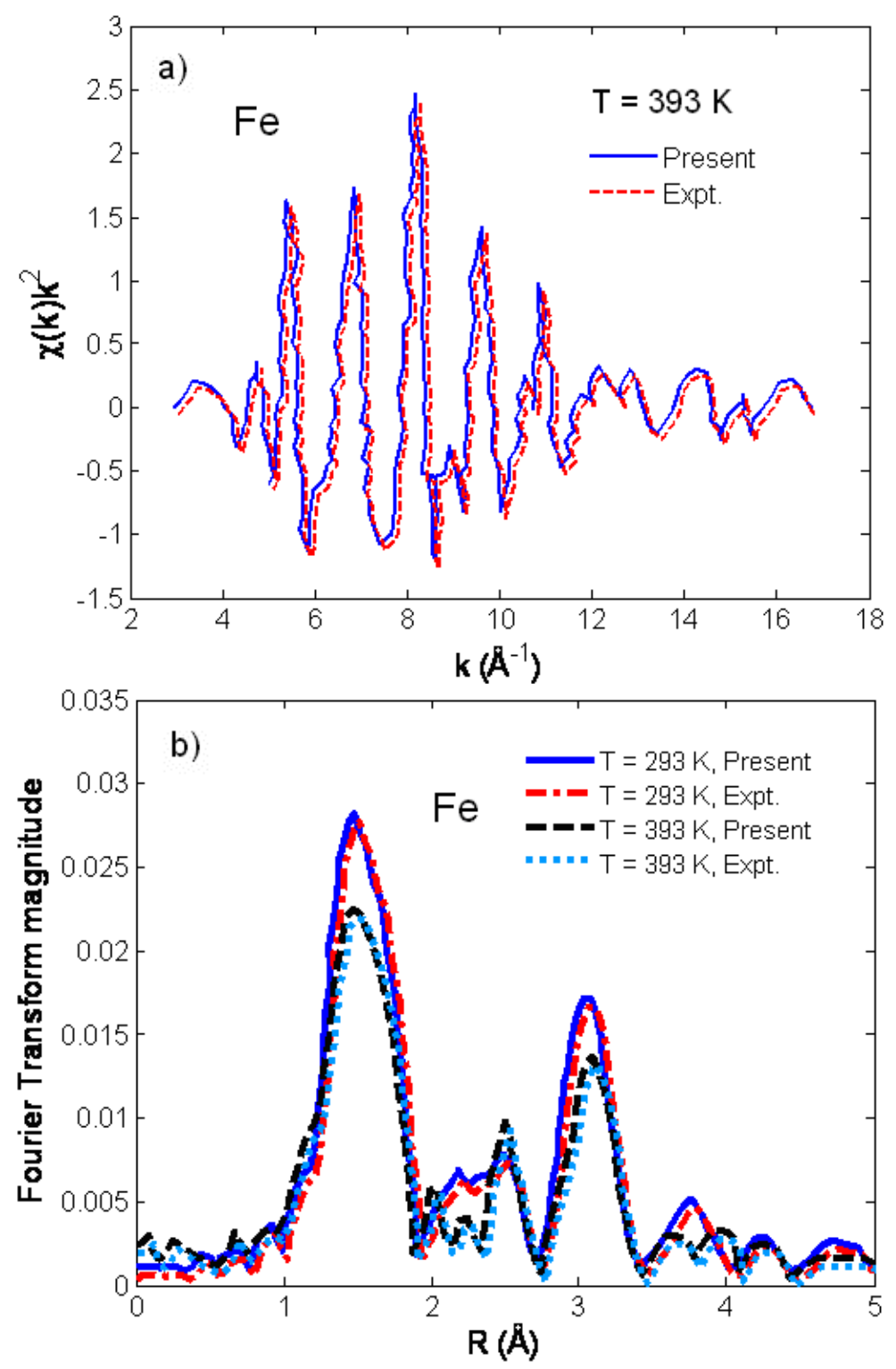

Fig. 4. a) XAFS spectra at $393 \mathrm{~K}$ and b) Fourier transform magnitudes of XAFS spectra at $293 \mathrm{~K}$ and $393 \mathrm{~K}$ for bcc phase of $\mathrm{Fe}$ calculated by the present theory including the above obtained cumulants in the comparison with the experimental data in [21].

Figures 4.a,b illustrate good agreement of XAFS spectra at $393 \mathrm{~K}$ and XAFS spectra Fourier transform magnitudes at $293 \mathrm{~K}$ and $393 \mathrm{~K}$ for bcc phase of Fe calculated using Eqs. (1) and (2) including the anharmonic contributions. The Fourier transform magnitudes are shifted when the temperature changes from $293 \mathrm{~K}$ to $393 \mathrm{~K}$ due to the anharmonic effects described by the cumulants obtained by the present theory. 


\section{CONCLUSIONS}

The anharmonic XAFS spectra and their Fourier transform magnitudes of bcc crystals have been studied using the ACDM high-order expanded Debye-Waller factors where the ACDM has been derived based on the MBPA and the FSNNCA. The obtained results contribute to valuation of the thermodynamic properties and anharmonic effects in XAFS that lead to getting the accurate information on structural and other parameters of bcc crystals taken from XAFS experiments.

The advantage and efficiency of the present theory in XAFS data analysis are illustrated by the good agreement of the numerical results for cumulants, XAFS spectra and their Fourier transform magnitudes for bcc phase of Fe with experiments. This makes it possible to reproduce the experimental XAFS data of bcc crystals using the present theory.

\section{ACKNOWLEDGEMENTS}

This research is funded by the Vietnam National Foundation for Science and Technology Development (NAFOSTED) under grant number 103.01-2015.10.

\section{REFERENCES}

[1] E. D. Crozier, J. J. Rehr and R. Ingalls, X-ray Absorption, edited by D. C. Koningsberger and R. Prins, Chap. 9, Wiley, New York, 1988.

[2] J. M. Tranquada and R. Ingalls, Phys. Rev. B 28 (1983) 3520.

[3] A. I. Frenkel and J. J. Rehr, Phys. Rev. B 48 (1993) 585.

[4] T. Miyanaga and T. Fujikawa, J. Phys. Soc. Jpn. 63 (1994) 1036; ibid. 3683.

[5] N. V. Hung and J. J. Rehr, Phys. Rev. B 56 (1997) 43.

[6] E. A. Stern, P. Livins, and Zhe Zhang, Phys. Rev. B 43 (1991) 8850.

[7] N. V. Hung and R. Frahm, Physica B 208 \& 209 (1995) 91.

[8] N. V. Hung, T. S. Tien, N. B. Duc and D. Q. Vuong, Mod. Phys. Lett. B 28 (2014) 1450174.

[9] T. Yokoyama, Phys. Rev. B 57 (1998) 3423.

[10] A. V. Poiarkova and J. J. Rehr, Phys. Rev. B 59 (1999) 948.

[11] N. V. Hung, N. B. Duc and R. R. Frahm, J. Phys. Soc. Jpn. 72 (2003) 1254.

[12] M. Daniel, D. M. Pease, N. Van Hung and J. I. Budnick, Phys. Rev. B 69 (2004) 134414.

[13] F. D. Vila, J. J. Rehr, H. H. Rossner and H. J. Krappe, Phys. Rev. B 76 (2007) 014301.

[14] N. V. Hung and P. Fornasini, J. Phys. Soc. Jpn. 76 (2007) 084601.

[15] N. V. Hung, L. H. Hung, T. S. Tien, and R. R. Frahm, Int. J. Mod. Phys. B 22 (2008) 5155.

[16] N. V. Hung, N. B. Trung, and B. Kirchner, Physica B 405 (2010) 2519.

[17] N. V. Hung, V. V. Hung, H. K. Hieu, R. R. Frahm, Physica B 406 (2011) 456.

[18] N. V. Hung, C. S. Thang, N. C. Toan, H. K. Hieu, Vacuum 101 (2014) 63.

[19] I. V. Pirog and T. I. Nedoseikina, Physica B 334 (2003) 123.

[20] N. V. Hung, T. T. Hue, H. D. Khoa, D. Q. Vuong, Physica B 503 (2016) 174.

[21] I. V. Pirog and T. I. Nedoseikina, Physica B 334 (2003) 123.

[22] G. D. Mahan, Many-Particle Physics, $2^{\text {nd }}$ ed., Plenum, New York, 1990.

[23] A. A. Maradudin, Dynamical Properties of Solids, ed. by G. K. Horton and A. A. Maradudin, Vol. 1, p. 1., North Holland, Amsterdam, 1974.

[24] N. V. Hung, T. T. Hue, N. B. Duc, VNUJ. Science 31 (2015) 23. 